\title{
La música como medio alternativo en la modificación de la conducta del niño durante el tratamiento dental
}

Cruz CM, Díaz-Pizán ME. La música como método alternativo en la modificación de la conducta del niño durante el tratamiento dental. Rev Estomatol Herediana 2005;15(1): 46 - 49

\section{RESUMEN}

El manejo de la ansiedad en un niño es un aspecto crítico del procedimiento dental. Por esta razón, muchas técnicas que envuelven métodos no farmacológicos han sido usadas para manejar la conducta del niño en el consultorio dental. El propósito del presente estudio fue evaluar el empleo de la música como una herramienta en la modificación de la conducta del niño, a fin de obtener un mayor grado de colaboración y satisfacción durante el tratamiento dental. La muestra estuvo constituida por 50 niños de 3 a 9 años de edad, todos ellos seleccionados al azar y atendidos en el área de Odontología Pediátrica Post-grado de la Clínica Estomatológica Central (CEC) de la Universidad Peruana Cayetano Heredia (UPCH). Todos los pacientes fueron diagnosticados con lesiones de caries dental y pulpitis irreversible. Fue un ensayo clínico de tipo comparativo. Los resultados no mostraron diferencias significativas en la conducta de los niños hacia el tratamiento dental, entre aquellos que escucharon música y aquellos que no lo hicieron. Los niños que recibieron música presentaron un mayor porcentaje de satisfacción del tratamiento.

Palabras clave: ANSIEDAD AL TRATAMIENTO ODONTOLÓGICO / MÚSICA / CONDUCTA Y MECANISMOS DE CONDUCTA.

Music as an alternative method to modify children's behaviour during dental treatment

ABSTRACT

Management of anxiety in a child is a critical aspect in dental procedures. For this reason, many techniques involving non-pharmacological methods have been used to manage the child's conduct in the dental office. The purpose of the present study was to evaluate the use of music as an appropriate tool to modify the child's conduct in order to obtain a better degree of collaboration and satisfaction during dental treatment. The sample of study was constituted by 50 randomly selected children from 3 to 9 years of age attending the Post-Graduate Pediatric Odontology Area of the Central Stomatologic Clinic of the Peruvian University Cayetano Heredia. All were diagnosed with carious lesions and irreversible pulpitis. The study was a clinical essay of comparative type. No statistical significant differences were found on the behaviour of children toward dental treatment between those who listened to music and those who did not. However, those children who listened to music presented a significantly higher percentage of treatment satisfaction.

Keywords: DENTAL ANXIETY / MUSIC / BEHAVIOR AND BEHAVIOR MECHANISMS.

\section{Carlos Miguel Cruz Chu ${ }^{1}$} María E. Díaz-Pizán ${ }^{2}$

Ex-Alumno de la Facultad de Estomatología. Docente del Departamento de Estomatología del Niño y el Adolescente. Facultad de Estomatología. Universidad Peruana Cayetano Heredia.

\section{Correspondencia}

Carlos Miguel Cruz Chú, Antonio Polo 744, Lima 21 - Perú. Telf: 97271958.

E-mail: correo_lima_peru@yahoo.com

Aceptado para publicación : 20 de junio del 2005

\section{Introducción}

El temor y la ansiedad frente al tratamiento dental son problemas comunes en niños y adolescentes. Generalmente, el paciente relaciona la ansiedad con el miedo al dolor. Las modernas técnicas de anestesia que utilizan los dentistas en la actualidad, si bien no han anulado el componente doloroso, por lo menos lo han reducido a niveles de aceptable tolerancia. Aún así, la ansiedad puede tornar al paciente hipersensible a los estímulos ejercidos sobre su cuerpo y, por lo tanto, sobre sus dientes; además, aumenta su estado de vigilia y tensión muscular (1).

El manejo de la conducta ansiosa en el niño es un aspecto crítico durante los procedimientos dentales. Por eso, en la actualidad, se utilizan diversas técnicas para manejar la conducta del paciente en el consultorio, estas técnicas pueden ser farmacológicas y no farmacológicas $(2,3)$.

Diferentes técnicas no farmacológicas han sido utilizadas a lo largo del tiempo para lograr una colaboración receptiva en el paciente. El "decir-mostrar-hacer" o "la hora del juego en el consultorio dental” se han reportado como métodos que motivan al niño a una mayor curiosidad y participación durante el procedimiento dental, evitando que éste muestre miedo (4). Otras alternativas como la "hipnosis" o el "manejo de la respiración”, se utilizan como distractores para que el niño deje de pensar en cosas negativas acerca del procedimiento dental, mientras le rea- lizan el tratamiento $(5,6)$

La utilización de la música en niños y adolescentes durante la práctica dental es considerada como una terapia de sugestión en donde el niño es animado a la fantasía. Lamentablemente, esta técnica no es muy utilizada probablemente por falta de información del clínico $(7,8)$.

Algunos investigadores han utilizado música ambiental, canciones infantiles, historias y cuentos; así como también, diversos temas de audición durante el tratamiento de niños pequeños o discapacitados, con la finalidad de lograr una mayor colaboración del paciente pediátrico $(9,10)$.

El propósito del presente estudio fue evaluar el empleo de la música como una herramienta apropiada en la modificación 
de conducta del niño, a fin de obtener un mayor grado de colaboración y satisfacción durante el tratamiento dental.

\section{Materiales y método}

La muestra de estudio estuvo constituida por 50 niños de 3 a 9 años de edad, seleccionados al azar, atendidos por los residentes del Área de Odontología Pediátrica Post-grado de la Clínica Estomatológica Central (CEC) de la Universidad Peruana Cayetano Heredia (UPCH). Todos ellos diagnosticados con lesiones de caries dental y pulpitis irreversible. La población se dividió en dos grupos: uno al que se le aplicó música y se denominó grupo de casos, y otro al cual no se aplicó música y sirvió como grupo control. La asignación de los pacientes a ambos grupos fue realizada en forma aleatoria. El diseño del estudio fue ensayo clínico de tipo comparativo.

La evaluación de la conducta de los pacientes se realizó utilizando la escala de clasificación de conducta de la Universidad del Estado de Ohio (11). El anotador, responsable de la observación y monitoreo del paciente, no tuvo conocimiento a que grupo pertenecía el niño (con música o sin música).

Una vez ubicado el niño en el sillón dental, se le colocó los audífonos conectados a un Walkman marca Sony ${ }^{\circledR}$ para que pudiera escuchar música de relajación antes de empezar el tratamiento, pero dependía del operador si el walkman estaba apagado o encendido; dando un tiempo de 2 a 8 minutos para que el niño se adecue a la música.

Terminado el tiempo de relajación, previo al tratamiento propiamente dicho, se evaluó la conducta del niño según la escala de clasificación de conducta de la Universidad del Estado de Ohio (11), para proceder a realizar el tratamiento dental. La conducta del paciente fue evaluada también en las diferentes etapas: al momento de colocar la anestesia, durante el aislamiento con dique de goma, al utilizar la pieza de mano de alta velocidad, y al momento de colocar el material restaurador. Luego del tratamiento dental propiamente dicho, se procedió a evaluar por última vez la conducta del niño, antes de retirarle los audífonos (Fig. 1).

Luego de salir el paciente del consultorio dental, respondió el test modificado de satisfacción y aceptabilidad del tratamiento de Tarnowski y Simonian (12) (Fig. 2). Al final de cada tratamiento el residente informaba al anotador, si el niño estuvo o no escuchando música a través de los audífonos. El mismo procedimiento fue realizado en ambos gru- pos (casos y controles) a fin de comparar sus respuestas. El volumen de música fue parcial (siendo 0 el mínimo y 10 el máximo), los niños tuvieron un volumen de 2,5 a 4, el cual podía variar según los requerimientos del operador.

El análisis de los datos incluyó la evaluación individual de las variables de estudio: conducta y satisfacción del tratamiento dental.

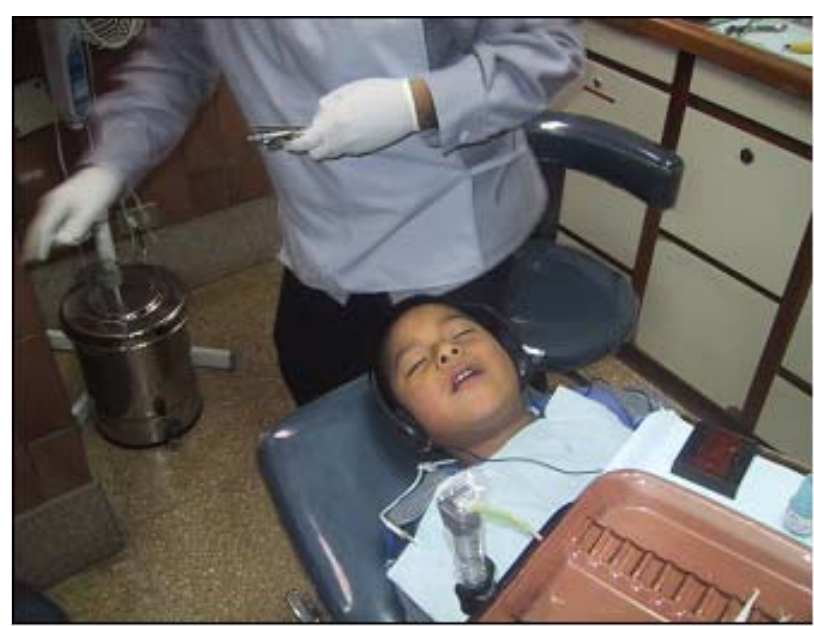

Figura 1. Niño que está recibiendo tratamiento odontológico, concentrado en la música

Me gustó que el dentista trabajara hoy día en mi diente

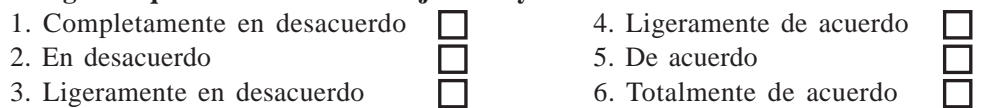

El tratamiento que recibí hoy día no me dolió

1. Completamente en desacuerdo

2. En desacuerdo

3. Ligeramente en desacuerdo

4. Ligeramente de acuerdo

5. De acuerdo

6. Totalmente de acuerdo

Disfruté mi visita al dentista hoy día

1. Completamente en desacuerdo

2. En desacuerdo

3. Ligeramente en desacuerdo

4. Ligeramente de acuerdo

5. De acuerdo

6. Totalmente de acuerdo

Me gustó como fui tratado hoy día

1. Completamente en desacuerdo

2. En desacuerdo

3. Ligeramente en desacuerdo

4. Ligeramente de acuerdo

5. De acuerdo

6. Totalmente de acuerdo

Me gustaría volver a ver al dentista de nuevo

1. Completamente en desacuerdo

2. En desacuerdo

3. Ligeramente en desacuerdo

4. Ligeramente de acuerdo

5. De acuerdo

6. Totalmente de acuerdo

Figura 2. Test modificado de satisfacción y aceptabilidad del tratamiento de Tarnowski y Simonian. 


\section{Resultados} de la evaluación de la conducta de los pacientes durante el procedimiento dental, según fueron expuestos o no a la música. La prueba U de Mann-Whiney, no arrojó diferencia significativa en ninguna de las situaciones ( $p>0,05)$. Al analizar los resultados de la conducta de los pacientes atendidos con música o sin música durante el tratamiento den-
La Tabla 1, muestra los resultados

tal, no se encontró diferencia estadística significativa y en casi todos los casos (más del 80\%) los niños permanecieron "quietos" durante la atención dental. Sin embargo, la Tabla 2, otorga una ventaja descriptiva al grupo de casos frente a los controles, en las respuestas del test modificado de satisfacción y aceptabilidad del tratamiento de Tarnowski y Simonian. Así se encontró que, el 56\% de los pacientes que escu-

Tabla 1. Conducta del paciente en los diferentes momentos del tratamiento dental según fue atendido con música o sin música.

\begin{tabular}{lllll}
\hline & \multicolumn{3}{l}{ con música $(\mathrm{n}=25)$} & \multicolumn{2}{c}{ sin música $(\mathrm{n}=25)$} \\
Períodos de Tratamiento & $\mathrm{n}$ & $\%$ & $\mathrm{~N}$ & $\%$ \\
\hline $\begin{array}{l}\text { Antes de empezar el tratamiento } \\
\text { Quieto }\end{array}$ & 22 & 88 & 24 & 96 \\
Movimiento & 3 & 12 & 1 & 4 \\
\hline Al colocar la anestesia & & & & \\
No Anesthesia & 11 & 44 & 12 & 48 \\
Quieto & 8 & 32 & 7 & 28 \\
Movimiento & 6 & 24 & 6 & 24 \\
\hline
\end{tabular}

\begin{tabular}{lllll}
\hline Aislamiento con dique de goma & & & & \\
Quieto & 20 & 80 & 18 & 72 \\
Movimiento & 5 & 20 & 7 & 28 \\
\hline Comenzando a usar pieza de alta & & & & \\
Quieto & 21 & 84 & 21 & 84 \\
Movimiento & 4 & 16 & 4 & 16 \\
\hline Al momento de colocar el material restaurador & 23 & 92 & 24 & 88 \\
Quieto & 2 & 8 & 3 & 12 \\
Movimiento & & & & \\
\hline Antes de retirarse del sillón dental & 23 & 92 & 25 & 100 \\
Quieto & 2 & 8 & 0 & 0 \\
Movimiento & & &
\end{tabular}

$(\mathrm{p}>0,05)$

Tabla 2. Frecuencia de respuestas al test modificado de satisfacción y aceptabilidad del tratamiento de Tarnowski y Simonian, según atención con música o sin música.

\begin{tabular}{|c|c|c|c|c|}
\hline \multirow[t]{2}{*}{ Pregunta del Test } & \multicolumn{4}{|c|}{ con música $(n=25)$} \\
\hline & $\mathrm{n}$ & $\%$ & $\mathrm{~N}$ & $\%$ \\
\hline \multicolumn{5}{|c|}{ Me gustó que el dentista trabajara hoy en mi diente } \\
\hline De acuerdo & 11 & 44 & 17 & 68 \\
\hline Totalmente de acuerdo* & 14 & 56 & 8 & 32 \\
\hline \multicolumn{5}{|c|}{ El tratamiento que recibí hoy día no me dolió } \\
\hline En desacuerdo & 5 & 20 & 11 & 44 \\
\hline De acuerdo & 6 & 24 & 9 & 36 \\
\hline Totalmente de acuerdo* & 14 & 56 & 5 & 20 \\
\hline \multicolumn{5}{|c|}{ Disfruté mi visita al dentista hoy día } \\
\hline De acuerdo & 11 & 44 & 15 & 60 \\
\hline Totalmente de acuerdo & 14 & 56 & 10 & 40 \\
\hline \multicolumn{5}{|c|}{ Me gustó cómo fui tratado hoy día } \\
\hline De acuerdo & 7 & 28 & 15 & 60 \\
\hline Totalmente de acuerdo* & 18 & 72 & 10 & 40 \\
\hline \multicolumn{5}{|c|}{ Me gustaría volver a ver al dentista de nuevo } \\
\hline En desacuerdo & 3 & 12 & 0 & 0 \\
\hline De acuerdo & 8 & 32 & 12 & 48 \\
\hline Totalmente de acuerdo & 14 & 56 & 13 & 52 \\
\hline
\end{tabular}

$* \mathrm{p}<0,05$ charon música manifestaron estar "totalmente de acuerdo" con la pregunta 1 del test: "me gustó que el dentista trabajara hoy día en mi diente”, mientras que en el grupo control la misma respuesta alcanzó el 32\%. Situación similar ocurrió con las respuestas a la pregunta 2 del test: "el tratamiento que recibí hoy día no me dolió”, el 56\% del grupo de casos y el 20\% del grupo control estuvo "totalmente de acuerdo", presentándose aquí una diferencia significativa $(\mathrm{p}<0,05)$. En el resto de las preguntas, no se hallo diferencia significativa entre los grupos de estudio ( $p>0,05)$.

\section{Discusión}

Aitken et al. (13) indican que la música no es una terapia eficaz para reducir la ansiedad ni el dolor dental, ni modifica la conducta negativa del paciente. Sin embargo, Corrêa y Guedes-Pinto (14) mencionan que la terapia con música crea un ambiente apropiado y favorable para el tratamiento dental del niño.

En el presente estudio los niños, que escucharon música durante el tratamiento, mostraron una conducta receptiva y de colaboración, similar a la que presentaron los niños que no fueron expuestos a la música (grupo control) pero que fueron tratados con otras técnicas no farmacológicas de modificación de la conducta. Estas técnicas han mostrado resultados positivos en la modificación de la conducta del niño $(8,15)$ teniendo como ventaja que son ampliamente aceptadas por los padres o tutores $(16,17)$.

Los resultados obtenidos en el presente estudio indican claramente que la música puede ser tan provechosa al igual que los otros métodos no farmacológicos. Sin embargo, se ha reportado que la música puede reducir la tensión y la ansiedad influyendo en el sistema nervioso, circulatorio y respiratorio; creando así un ambiente tranquilo para el niño en el consultorio dental (14) y mostrando que la terapia con música es la mejor opción.

La terapia con música es simple de utilizar y fácil de aplicar, no requiere de equipo sofisticado que implique altos costos para el profesional y el paciente. Reinhold (10) recomienda que para utilizar la música en la modificación de con 
ducta se requiere un «ambiente cerrado" para aislar al niño de sonidos externos. De lo cual se concluye que el uso de los walkmans no sería lo adecuado. Sin embargo el presente estudio demostró, que éste sistema de aislamiento parcial permitió al niño concentrarse en la música y al mismo tiempo oír las instrucciones del dentista. Esto es importante, puesto que la comunicación con el dentista es esencial para que el niño colabore durante el experiencia dental (18).

Con respecto al grado de satisfacción durante el tratamiento dental, se encontró que más de dos tercios de los niños se divirtieron en la visita dental y no sintieron dolor. Esto indica que la música tiene un efecto positivo en la actitud del niño hacia el tratamiento dental. Bustillo et al. (19) al atender a 100 niños usando música, encontraron que el 92\% de ellos presentaron una conducta excelente, mientras que solamente el $6 \%$ presentaron una conducta aceptable. Con estos resultados concluyeron que, la música es un método alternativo importante para modificar la conducta del paciente pediátrico.

Una limitación encontrada en el presente estudio fue que los odontopediatras no están familiarizados con el uso de la música durante el tratamiento, y requieren de un cierto entrenamiento antes de su aplicación. El estudio realizado por Silva y Reis (20) en el Brasil corrobora este hecho y sugieren un mayor empleo de la técnica odontológica con música.. Se sugiere que los dentistas deberían estar bien informados y entrenados en esta técnica, así como también de las canciones infantiles más populares del momento. En éste estudio, todos los temas musicales fueron elegidos de las series infantiles de mayor sintonía en nuestro medio. Por ejemplo, las canciones que se relacionaban con la valentía de una persona fueron utilizadas cuando se le aplicaba la anestesia al niño.

Sugerimos que se debería de fomentar la difusión de la música como método alternativo en la modificación de la conducta del niño, sobre todo a nivel de postgrado, ya que son los futuros especialistas quienes deberían dominar todas las formas de manejo de conduc- ta, sobre todo si el paciente va a sentir confort y satisfacción. Se deberían realizar también, estudios complementarios utilizando la música como terapia de tratamiento, desde la primera visita hasta el final; ello nos ayudaría a evaluar mejor los efectos de la música en odontología infantil.

\section{Conclusiones}

- La música es un método alternativo en el manejo de la conducta de niños durante el tratamiento dental.

- La música permite un nivel aceptable de colaboración del paciente puesto que hace que el niño enfrente de mejor forma las situaciones que causan ansiedad .

- La música brinda al paciente niño un mayor grado de satisfacción hacia el tratamiento dental.

\section{Referencias bibliográficas}

1. Blatto B, Vismara V. Medicina natural en odontología. Tratamiento de la ansiedad. Journal de Clínica en Odontología 1999/2000;15(5):46-54.

2. Glasrud P. Dentists' characteristics and child behavior management techniques. J Dent Child 1984;51:337-43.

3. Edmunds D, Rosen M. Management of anxiety in dental practice in the UK. Brit Dent J 1989;166:253-8.

4. Martí S, Pirrone A, Levinzon G, Breuss M, Penucci A. La hora del juego en la atención odontológica de niños. Boletín de la Asociación Argentina de Odontología para Niños 1998;28(2):18-21.

5. Gokli M, Wood A, Mourino A, Farrington F, Best A. Hypnosis as an adjunct to the administration of local anesthetic in pediatric patients. J Dent Child 1994;61:272-5.

6. Levitt J, McGoldrick P, Evans D. The management of severe dental phobia in an adolescent boy: a case report. Int J Paediatr Dent 2000;10:348-53.

7. Shaw A, Niven N. Theoretical concepts and practical applications of hypnosis in the treatment of children and adolescents with dental fear and anxiety. Brit Dent J 1996;180:11-6.

8. Clive F, Mc Murray N, Giebartowski J. Strategies used by dentists in
Victoria, Australia, to manage children with anxiety or behavior problems. J Dent Child 1991;58: 223-8.

9. Di Nasso P. Cuidado dental del niño con discapacidad. Boletín de la Asociación Argentina de Odontología para Niños 20012002;30(4):21-4.

10. Reinhold M. Control del miedo y del dolor en el tratamiento estomatológico en la infancia mediante audioanalgesia. Quintessence (Sp ed.) 1994;7(3): 188-91.

11. Wilson S, Molina L, Preisch J, Weaver J. The effect of electronic dental anesthesia on behavior during local anesthetic injection in the young, sedated dental patient. Pediatr Dent 1999; 21(1):12-7.

12. Tarnowski KJ, Simonian SJ. Assessing treatment acceptance: The abbreviated Acceptability Rating Profile. J Behav Ther Exp Psy 1992; 23:101-6.

13. Aitken J, Wilson S, Coury D, Moursi A. The effect of music distraction on pain, anxiety and behavior in pediatric dental patients. Pediatr Dent 2002;24:114-8.

14. Corrêa MS, Guedes-Pinto CA. Técnicas psicológicas utilizadas en odontopediatría. En: Odontopediatría. Guedes-Pinto CA. $7^{\text {th }}$ ed. 2003. Libraria Santos Editora Ltda., São Paulo.

15. Rosemberg H. Behavior Modification for the child dental patient. J Dent Child 1974; March-April: 31-34.

16. Scott S, García-Godoy F. Attitudes of Hispanic parents toward behavior management techniques. J Dent Child 1998:65;128-31.

17. Peretz B, Zadik D. Parents’ attitudes toward behavior management techniques during dental treatment. Pediatr Dent 1999;21:201-4.

18. Pinkham J. Linguistic maturity as a determinant of child patient behavior in the dental office. J Dent Child 1997;64:322-6.

19. Bustillo G, Guedes Pinto AC, Sagretti O. Influencia da música no tratamento odontopediátrico. Rev Assoc Paul Cir Dent 1992; 46(2):731-4.

20. Silva E, Reis C. Utilização da música no consultorio odontológico. RGO 1994;42(2):116-8. 